\title{
The impact of admission to a pediatric intensive care unit assessed by means of global and cognitive performance scales
}

\author{
Patrícia T. Alievi, ${ }^{1}$ Paulo R. A. Carvalho, ${ }^{2}$ Eliana A. Trotta, ${ }^{3}$ Ricardo Mombelli Filho $^{4}$
}

\begin{abstract}
Objective: To assess the impact of admission to the pediatric intensive care unit (ICU) at the Hospital de Clínicas de Porto Alegre, RS, Brazil on children's cognitive and global performance.

Methods: An observational, longitudinal study of a sequential sample of critically ill children. The following indicators were used: the Pediatric Index of Mortality (PIM), for severity and risk of death at admission, the Pediatric Cerebral Performance Category (PCPC), for cognitive morbidity and the Pediatric Overall Performance Category (POPC), for global morbidity, at admission and at discharge. Morbidity related to the ICU was measured according to the difference between classifications at discharge and at admission (delta scores). The Kruskal-Wallis test was applied.
\end{abstract}

Results: A total of 443 patients were assessed, $54 \%$ of whom were male, with a median age of 12 months (IQ 4-45), and a median ICU stay of 4.24 days (IQ 2.4-8). The mortality rate was $6.3 \%$. The median PIM score was $2.36 \%$ (IQ 1-7). On admission, $46 \%$ of the patients had some degree of cognitive morbidity and $66 \%$ had some degree of global morbidity. At discharge there was $60 \%$ cognitive morbidity and $86 \%$ global morbidity. The assessment of ICUrelated morbidity revealed that $25 \%$ of the patients had undergone cognitive changes while $41 \%$ had undergone global variations, at discharge compared with admission.

Conclusions: Although affected by the elevated degree of morbidity at admission, the impact of the ICU stay was more significant in the global than in the cognitive domain. In the same manner, both risk of death at admission and length of stay had a significant effect on the morbidity of severely ill patients.

J Pediatr (Rio J). 2007;83(6): 505-511: Outcome, critical care, pediatric cerebral performance category, pediatric overall performance category, morbidity.

\section{Introduction}

In critical care medicine, intensive care unit (ICU) results are assessed almost exclusively on the basis of the outcomes "death" or "survival," by means of indicators such as mortality rate, readmission rate or the rates of complications related to certain treatments. Indicators of morbidity are important aids, ${ }^{1}$ but can be difficult to quantify, particularly in children. ${ }^{2}$
In pediatric intensive care, however, death may be a relatively rare event, depending on the volume of patients admitted and the size and type of unit, and does not therefore allow for the full extent of repercussions provoked by the acute disease and by the stay in ICU to be assessed. ${ }^{3-5}$ Admission to and treatment in intensive care may not result in death, but in varying degrees of incapacity among survivors. ${ }^{4}$

1. Mestre. Pediatra rotineira, Emergência Pediátrica, Hospital da Criança Santo Antônio, Complexo Hospitalar Santa Casa, Porto Alegre, RS, Brazil.

2. Doutor. Professor associado, Departamento de Pediatria e Puericultura e Programa de Pós-Graduação em Ciências Médicas: Pediatria, Faculdade de Medicina, Universidade Federal do Rio Grande do Sul (UFRGS), Porto Alegre, RS, Brazil.

3. Mestre. Professora adjunta, Departamento de Pediatria, UFRGS, Porto Alegre, RS, Brazil.

4. Médico.

No conflicts of interest declared concerning the publication of this article.

Suggested citation: Alievi PT, Carvalho PR, Trotta EA, Mombelli Filho R. The impact of admission to a pediatric intensive care unit assessed my means of global and cognitive performance scales. J Pediatr (Rio J). 2007;83(6):505-511. 
Evaluation of the results of medical interventions can better quantify the efficacy of different treatments, making possible to take better decisions, to standardize conduct and to optimize resource utilization. ${ }^{6,7}$ This becomes more relevant to the extent that it is unclear what impact ICU stays have on patients' physiological and cognitive functions. Assessment of the level of patients' cognitive and functional impairment after a period of significant stress and of their potential need for help to reenter the family and social environments can both be of help to better understanding the repercussions of acute disease or trauma and of medical care. ${ }^{8}$

While there are many studies of health condition and instruments for measuring quality of life in adults, ${ }^{9}$ few instruments have been validated for use with children. ${ }^{4}$ The majority of these only measure the degree of incapacity with questions on recovery or development.

Fiser et al. have reported on the validation studies of two new outcome scales for children, the Pediatric Cerebral Performance Category (PCPC) and the Pediatric Overall Performance Category (POPC). ${ }^{2,10}$ These are two of the rare instruments that can be used with children less than 1 year old. ${ }^{3}$ These two indices were adapted for pediatric use, since, in essence, they were already being used to evaluate adults. ${ }^{11}$ Variations in scores on these scales exhibited a direct relationship with the degree of morbidity measured by other already established indices, such as length of stay in the ICU, total hospital costs, post-discharge home care and disease severity, in an analysis of 1,469 patients admitted to Pediatric ICU. ${ }^{2}$ The difference between the categories at discharge and at admission was defined as the delta score, being the most direct reflection of the change in functional capacity resulting from a disease episode or injury. A multicenter study of 16 intensive care units in the United States investigated 11,104 patients using the PCPC and POPC scales, reproducing and validating the results of the initial study. ${ }^{12}$

These scales have also been recommended for relating results associated with cardiopulmonary resuscitation in children ${ }^{13,14}$ and with neurological outcomes in head traumas. ${ }^{15,16}$

The objective of this study was to evaluate the impact of admission on the cognitive and functional performance of children admitted to the Pediatric ICU at the Hospital de Clínicas de Porto Alegre (HCPA), by means of application of the POPC and $P C P C$ at admission and at discharge to obtain a delta score, in addition to relating it to the Pediatric Index of Mortality (PIM) at admission ${ }^{17}$ and with length of stay in the ICU.

\section{Methods}

This was a longitudinal, observational and individual study, involving minimum risk, of a sequential sample of the patients admitted to the Pediatric ICU at the HCPA, during the periods between April and November of 2002 and between June 2004 and January 2005. Patients were excluded if they spent less than 24 hours in the ICU or if their parents and/or guardians did not agree to their participation in the study. General data collected were: age, sex, diagnosis at admission, origin, length of ICU stay, PIM on admission and outcome.

The HCPA is a general, university hospital, offering tertiary care, with a 13-bed pediatric ICU that admits patients aged 1 month to 17 years with clinical or surgical conditions, except for cases of trauma and heart surgery. It has a yearly average of 550 admissions. This Pediatric ICU is a referral center for critically ill children, receiving patients from all over the state of Rio Grande do Sul.

Transcultural adaptation was carried out on the research instruments (PCPC and POPC) by means of translation into Brazilian Portuguese with validation by back-translation. ${ }^{18}$

Morbidity was assessed by means of use of the PCPC and POPC scales applied at admission, by means of clinical history taken from parents, and, at patient discharge, by means of clinical observation. The measurement employed to evaluate the impact of the ICU stay was the morbidity classification, i.e., at discharge and at admission - the delta score (deltaPCPC and delta-POPC).

It should be emphasized that a delta score of zero indicates that a child's performance did not alter during their time in the ICU. A positive value signifies an increase in morbidity, and a negative value indicates that there has been an improvement with relation to the pre-admission state.

Clinical history was taken by one of the authors (P.T.A.), who also observed all of the patients studied. Given the potential subjectivity when applying scales, a pilot study was carried out with 30 patients to assess interobserver agreement, with two independent, blinded observers, and which returned a good level of agreement (kappa index of 0.7 to 1.0 ).

The sample size was calculated as 432 patients, with a power of $80 \%$ and alpha $=0.05$, PEPI, version 2 software.

Quantitative variables were described using means and standard deviations when distribution was symmetrical, and medians and interquartile ranges when asymmetrical. The Kruskal-Wallis test was used to compare length of ICU stay and PIM according to delta scores. The level of significance was set at 5\%. Data were analyzed using SPSS, version 14.0.

The research project was approved by the Research Ethics Committee at the HCPA. Parents and guardians were asked to agree to participation by means of signing a free and informed consent form.

\section{Results}

A total of 499 patients were considered eligible during the study period. Fifty- four patients were excluded because they spent less than 24 hours in the ICU and two because their guardians did not agree to their participation. Therefore, including both periods, a total of 443 patients were studied. 
The characteristics of the samples for each period were compared and no differences were observed between them. The general characteristics of the whole sample are listed in Table 1.

The most frequent causes of admission were respiratory dysfunction $(43.3 \%)$, sepsis and shock $(25.5 \%)$, postoperative care (19\%) and convulsive crisis (8.1\%).

On admission, some degree of cognitive morbidity was identified in $46 \%$ of the children and some degree of functional morbidity was detected in $66 \%$, according to the classification categories employed for this study. At discharge, $60 \%$ of the children had some degree of cognitive morbidity and $86 \%$ had functional morbidity. Figure 1 illustrates the outcomes of each group of patients in each of the PCPC and POPC categories at admission in relation to their PCPC and POPC categories at discharge.

The median cognitive category (PCPC) at admission was 1 (normal) and, at discharge it was 2 (mild disability). The median functional category (POPC) at admission was 2 (mild overall disability) and at discharge it was 3 (moderate overall disability).

Table 2 demonstrates the impact of the ICU stay, indicated by delta scores, for both cognitive categories (PCPC)

Table 1 - General characteristics of the sample

\begin{tabular}{lc} 
Sex & \\
Male & $241(54.4 \%)$ \\
Female & $202(45.6 \%)$ \\
Age & 12 months $(4-45)$ \\
Origin & \\
Other hospitals & $140(31.6 \%)$ \\
Wards & $130(29.3 \%)$ \\
Emergency & $108(24.4 \%)$ \\
Surgical department & $53(12 \%)$ \\
Outpatient department & $12(2.7 \%)$ \\
Length of ICU stay & 4.24 days $(2.4-8)$ \\
PIM & $2.36 \%(1-7)$ \\
Outcome & \\
Wards & \\
Death & $404(91.2 \%)$ \\
Transferred to another & $28(6.3 \%)$ \\
Home & \\
\hline
\end{tabular}

Results expressed in absolute numbers (percentages), except for age, expressed in months, length of hospital stay, in days and PIM (Pediatric Index of Mortality), expressed in percentages (medians and interquartile range).

$\mathrm{PIM}=$ pediatric index of mortality; ICU = intensive care unit. and functional categories (POPC). In 75\% of cases, the deltaPCPC score exhibited no change at discharge compared with admission (delta $=0$ ), while the delta-POPC score indicated no variation in $59 \%$ of cases.

Table 3 illustrates the relationships between ICU length of stay and risk of mortality and the delta-PCPC and delta-POPC scores.

There was an improvement in global function, according to the POPC, in a subset of 21 patients (4.7\%), 85.7\% of whom were admitted for postoperative care. This group had the lowest risk of mortality.

The statistical analysis indicated significant differences between delta scores and ICU length of stay $(p<0.001)$ and between delta scores and risk of mortality $(p<0.001)$.

With the exception of delta score 5 , the risk of mortality increased in line with increases in delta-POPC score, i.e. as function declined. The increase in hospital length of stay was not linear, with patients with delta- PCPC and delta-POPC scores of 2 and 3 having the longest median stays.

\section{Discussion}

In this study, we identified some degree of cognitive disability in $46 \%$ of the patients at admission, and functional disability in approximately $67 \%$ of them. In comparison with other published studies that have employed the same methodology, our data exhibit similar results, although of greater magnitude. In the original study by Fiser, where the scales were applied to 1,469 children, $16 \%$ were unhealthy at the time of admission in terms of cognitive performance and $43 \%$ had abnormal functional performance. ${ }^{2}$ In work published by Oom, who studied 580 patients, abnormal cognitive performance was identified in $24 \%$ and functional problems in $49 \%$ at the time of admission to an ICU. ${ }^{4}$ In the multicenter study by Fiser et al., which enrolled 11,104 patients, the figures were closer to our results, with $31 \%$ of the patients presenting with some degree of cognitive morbidity at admission and 59\% with functional morbidity. ${ }^{12}$ Volakli et al., in a study evaluating mortality and morbidity among patients 2 years after admission to an ICU, found that $25.8 \%$ of the patients had already presented alterations according to the PCPC scale and $40 \%$ by the POPC at the time of admission. ${ }^{19}$ Cunha et al. studied functional status of children before and after spending time in Portuguese pediatric intensive care units, reporting cognitive morbidity in $28 \%$ and functional morbidity in $43 \%$ of the patients. ${ }^{20}$ Gemke et al. employed at different instruments and observed damage to overall health in $68.5 \%$ of the patients prior to admission. ${ }^{3}$

In our study, the assessment of patients at the time of discharge from the ICU found some degree of cognitive damage in $60 \%$ of children and functional damage in $85 \%$. The studies carried out by Fiser reported rates of cognitive alterations varying from de 15 to $42 \%$ and rates of functional alterations varying from 70 to $73 \% .^{2,12}$ In the study undertaken by Oom, 


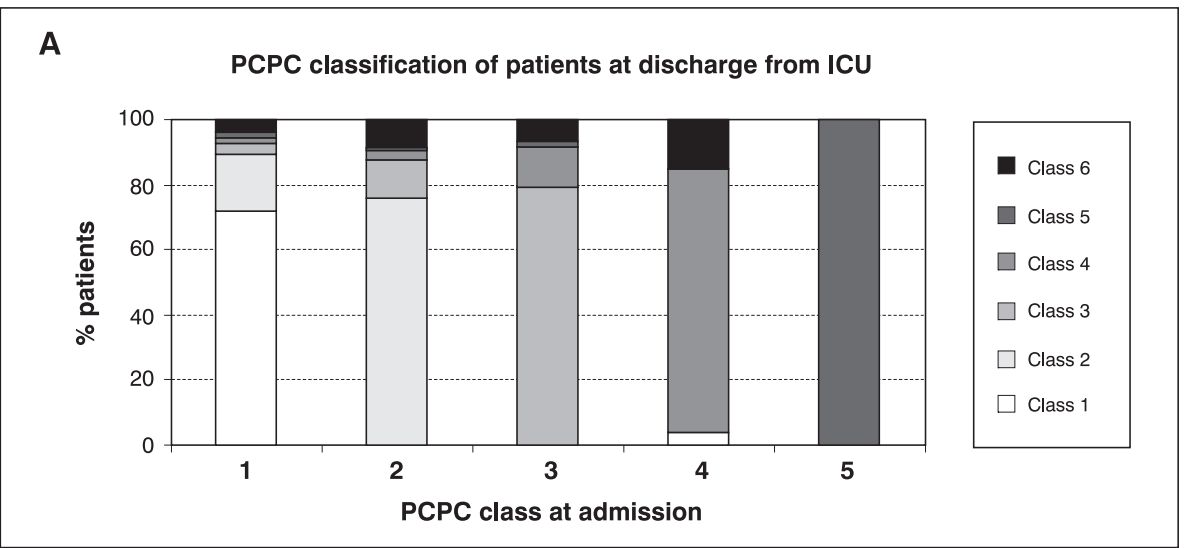

\section{B}

POPC classification of patients at discharge from ICU

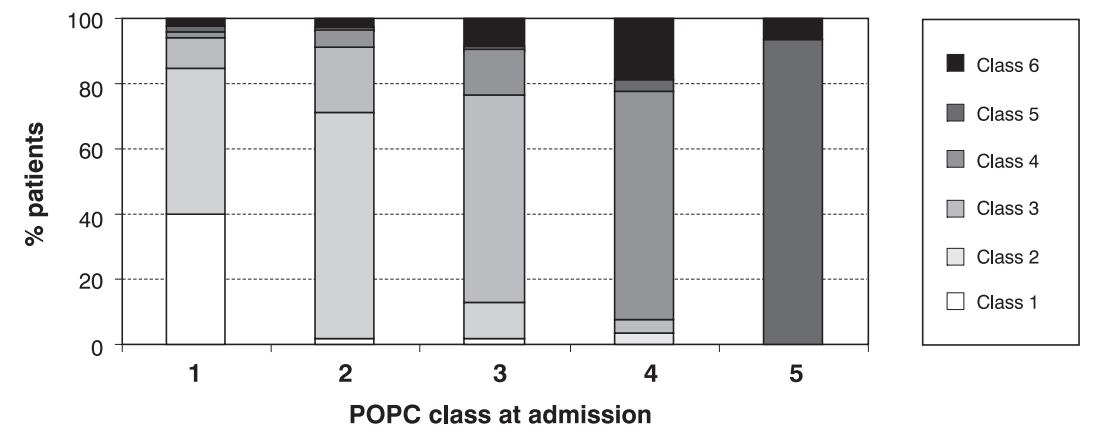

$\mathrm{PCPC}=$ pediatric cerebral performance category; $\mathrm{POPC}=$ pediatric overall performance category; $\mathrm{ICU}=$ intensive care unit.

Figure 1 - A) PCPC classification of the patients at admission and at discharge. B) POPC classification of the patients at admission and at discharge

the presence of cognitive sequelae was observed in $32 \%$ of the children and of physical sequelae in $62 \%$ at discharge. ${ }^{4}$ However, Cunha et al. reported that $34 \%$ of their patients exhibited cognitive damage and $53 \%$ functional damage. ${ }^{20}$ Taylor et al. assessed functional results after 3 years of followup, detected that $10.3 \%$ of the survivors had suffered unfavorable outcomes and would probably depend on the care of others, while $89.7 \%$ had had favorable results and would probably live an independent life. ${ }^{21}$

The delta-PCPC and delta-POPC scores represent a more direct perspective on the changes to functional and cognitive capacities resulting from an acute disease episode and/or an ICU stay. Therefore, according to Oom, irrespective of a greater or lesser extent of morbidity at the time of discharge, the impact of the acute disease and the ICU stay can only be assessed quantitatively by summing morbidity suffered by patients individually, by means of delta scores. ${ }^{4}$ In this study we observed worsened cognitive function in $25 \%$ of the children and worsened functional performance in approximately
Table 2 - Distribution of delta-PCPC and delta-POPC scores

\begin{tabular}{lccc}
\hline delta-PCPC & Frequency & delta-POPC & Frequency \\
\hline-3 & $1(0.2)$ & -1 & $21(4.7)$ \\
0 & $332(74.9)$ & 0 & $261(58.9)$ \\
1 & $62(14.0)$ & 1 & $107(24.2)$ \\
2 & $15(3.4)$ & 2 & $31(7.0)$ \\
3 & $10(2.3)$ & 3 & $14(3.2)$ \\
4 & $13(2.9)$ & 4 & $5(1.1)$ \\
5 & $10(2.3)$ & 5 & $4(0.9)$ \\
Total & $443(100)$ & Total & $443(100)$
\end{tabular}

$\mathrm{PCPC}=$ pediatric cerebral performance category; $\mathrm{POPC}=$ pediatric overall performance category. Frequencies are given as absolute values with their respective percentages in parentheses. 
Table 3 - Length of ICU stay and PIM in relation to delta scores

\begin{tabular}{|c|c|c|c|c|c|c|}
\hline \multirow[b]{2}{*}{ Delta score } & \multicolumn{3}{|c|}{ PCPC } & \multicolumn{3}{|c|}{ POPC } \\
\hline & $\mathbf{n}$ & $\begin{array}{l}\text { ICU length of stay } \\
\text { (days) }\end{array}$ & $\begin{array}{l}\text { PIM } \\
\text { (\%) }\end{array}$ & $\mathbf{n}$ & $\begin{array}{l}\text { ICU length of stay } \\
\text { (days) }\end{array}$ & $\begin{array}{l}\text { PIM } \\
(\%)\end{array}$ \\
\hline $\begin{array}{l}\text { Improvement } \\
(\text { delta }<0)\end{array}$ & 1 & - & - & 21 & $3.78(5.82-9.57)^{\mathrm{a}}$ & $1.5(0.7-2)^{\mathrm{a}}$ \\
\hline $\begin{array}{l}\text { No change } \\
(\text { delta }=0)\end{array}$ & 332 & $3.92(2.19-7.05)^{a}$ & $1.7(1-5)^{a}$ & 261 & $3.59(1.96-5.89)^{a}$ & $1(1-5)^{a}$ \\
\hline $\begin{array}{l}1 \text { category } \\
\text { worse } \\
(\text { delta }=1)\end{array}$ & 62 & $7.05(4.21-11.54)^{\mathrm{b}}$ & $4(1-9)^{b}$ & 107 & $6.20(3.87-9.56)^{b}$ & $3(1-8)^{\mathrm{ab}}$ \\
\hline $\begin{array}{l}2 \text { categories } \\
\text { worse } \\
(\text { delta }=2)\end{array}$ & 15 & $6.06(2.22-18.22)^{\mathrm{ab}}$ & $10(6-20)^{b}$ & 31 & $5.87(2.94-12.98)^{\mathrm{b}}$ & $9(3-20)^{\mathrm{b}}$ \\
\hline $\begin{array}{l}3 \text { categories } \\
\text { worse } \\
(\text { delta }=3)\end{array}$ & 10 & $7.61(3.17-16.91)^{\mathrm{ab}}$ & $8(3-27)^{b}$ & 14 & $5.75(3.27-18.71)^{\mathrm{ab}}$ & $17(3-36)^{b}$ \\
\hline $\begin{array}{l}4 \text { categories } \\
\text { worse } \\
(\text { delta }=4)\end{array}$ & 13 & $4.11(2.01-8.76)^{\mathrm{ab}}$ & $26(6-32)^{b}$ & 5 & $2.62(1.73-11.34)^{\mathrm{ab}}$ & $28(12-64)^{\mathrm{b}}$ \\
\hline \multirow[t]{2}{*}{$\begin{array}{l}5 \text { categories } \\
\text { worse } \\
(\text { delta }=5)\end{array}$} & 10 & $3.13(2.39-20.53)^{\mathrm{ab}}$ & $11(4-36)^{b}$ & 4 & $4.58(2.55-46.33)^{\mathrm{ab}}$ & $4(2-60)^{a}$ \\
\hline & & $p<0.001$ & $p<0.001$ & & $p<0.001$ & $p<0.001$ \\
\hline
\end{tabular}

$\mathrm{PCPC}=$ pediatric cerebral performance category; POPC = pediatric overall performance category; PIM = pediatric index of mortality. Results expressed as medians and interquartiles; Kruskal-Wallis test. Medians followed by the same letter in the vertical are statistically equal according to the Tukey test.

$37 \%$ of them. These data denote that these patients have been compromised to a greater extent than patients in other studies with similar methodology. Fiser et al. observed increased cognitive and functional morbidity in 14 and $24 \%$ of cases, respectively, ${ }^{14}$ and Oom in 10 and $20 \%$, respectively. ${ }^{4}$ Cunha et al. observed even lower rates, of 11 and $16 \% .^{20}$

On the other hand, cases of improvement are also reported, as in this study, where we observed 21 children with a negative delta-POPC, accounting for $4.7 \%$ of cases. For example, Cunha et al. observed $7 \%$ of cases with functional improvement. ${ }^{20}$ In the original study carried out by Fiser, just four cases had functional improvement. ${ }^{2}$ However, in the study involving 11,104 patients, 576 patients improved, accounting for $5.1 \%$ of cases. ${ }^{12}$ Oom also found similar results, reporting $6.4 \%$ of cases with negative delta-POPC. ${ }^{4}$ In common with this study, the overwhelming majority of functional improvement cases were patients in postoperative and liver or kidney post-transplantation care, i.e., patients whose disabilities and/or diseases have been repaired and/or improved by means of surgical operations. ${ }^{2,4,12}$

One thing which attracts attention in this study is a large percentage of patients who arrived at the ICU with some degree of compromise, whether cognitive or global.

This means that they could not be assessed without knowing their pre-morbid state, since at discharge any dysfunction or disability identified would be erroneously imputed to the ICU treatment or the disease which caused admission. At discharge, the patients left the ICU with significant degrees of morbidity, many with worsened function. These results lead to reflections on what types of patients most benefit from intensive care and on how patients considered chronic or with incurable sequelae are being treated, since these facts are related with the high demand of critical patients and the constant shortage of ICU beds. Therefore, a wide-ranging discussion on admission and discharge criteria, on aggression and 
treatment limitation and on quantity and quality of survival should take place in order to attempt to avoid or minimize these problems. ${ }^{22,23}$

Certain features and factors which may have been related with the higher numbers observed during this study, in other words, the greater the integers patients with morbidity, are the characteristics of the ICU at the HCPA and of the patients treated there. It is a tertiary hospital, with a high level of modern technology and is a reference centre for cancer patients, for people with rare genetic syndromes and inborn errors of metabolism and for patients with the most diverse range of neurological diseases. Therefore, it is a unit which treats high risk patients and where more aggressive treatments are available, which often results in lower mortality but higher morbidity among these patients. In the study by Oom, ${ }^{4}$ there was also a higher percentage of children with altered PCPC at admission, which was also explained by the author as a result of a high prevalence of patients with neurological diseases.

Aspects which could be the cause of measurement bias or limitations to this study are the fact that the scales have a certain characteristic of subjectivity in terms of their application and the fact that morbidity at admission was classified retrospectively. The inter-observer reliability test performed during the pilot study was an important issue and demonstrated, as has been demonstrated in other studies, that the use of these scales is possible and reliable for the assessment of patients. ${ }^{12}$ Therefore, this could be a method for institutions to adopt when they wish to monitor their performance and also for comparison of the results among ICUs.

The ideal moment at which to take measurements has not been well defined. Assessment at the end of a period of treatment, at discharge from the ICU, as was performed here, is the most frequently described method. ${ }^{24}$ This however, does not provide information on long-term results, and very often underestimates their degree of recovery. Over the course of years, many will continue to have disabilities, even more so if they do not receive outpatients follow-up adequate to their requirements, but also many others will improve and even overcome their limitations. Therefore, assessment at ICU discharge is of fundamental importance in order to know what these requirements are, serving as a tool with which to investigate specific difficulties and, in this way, aid in their recovery and it is desirable that they receive follow-up care and stimulation over the long term.

Despite suffering the influence of an elevated rate of morbidity at admission, the impact of an ICU stay was more significant in the global domain than in the cognitive area. Furthermore, both severity of the disease at admission and the length of stay have a significant effect on the morbidity of critically ill patients.

\section{References}

1. Morrison AL, Gillis J, O'Connell AJ, Schell DN, Dossetor DR, Mellis C. Quality of life of survivors of pediatric intensive care. Pediatr Crit Care Med. 2002;3:1-5.
2. Fiser DH. Assessing the outcome of pediatric intensive care. J Pediatr. 1992; 121:68-74.

3. Gemke RJ, Bonsel GJ, van Vught AJ. Long term survival and state of health after paediatric intensive care. Arch Dis Child. 1995; 73:196-201.

4. Oom P. Morbilidade em cuidados intensivos pediátricos. Acta Pediatr Port. 2004;35:279-85.

5. Marcin JP, Song J, Leigh JP. The impact of pediatric intensive care unit volume on mortality: a hierarchical instrumental variable analysis. Pediatric Crit Care Med. 2005;6:136-41.

6. Epstein AM. The outcomes movement - will it get us where we want to go? N Engl J Med. 1990;323:266-70.

7. Fiser DH. Outcome evaluations as measures of quality in pediatric intensive care. Pediatr Clin North Am. 1994;41:142338.

8. Gordon SM, Jackson JC, Ely EW, Burger C, Hopkins RO. Clinical identification of cognitive impairment in ICU survivors: insights for intensivists. Intensive Care Med. 2004;30:1997-2008.

9. Hurel D, Loirat P, Saulnier F, Nicolas F, Brivet F. Quality of life 6 months after intensive care: results of a prospective multicenter study using a generic health status scale and a satisfaction scale. Intensive Care Med. 1997;23:331-7.

10. Fiser DH, Long N, Roberson PK, Hefley G, Zolten K, BrodieFowler M. Relationship of pediatric overall performance category and pediatric cerebral performance category scores at pediatric intensive care unit discharge with outcome measures collected at hospital discharge and 1- and 6-month follow-up assessments. Crit Care Med. 2000;28:2616-20.

11. Jennett $B$, Bond M. Assessment of outcome after severe brain damage. Lancet. 1975;1:480-4.

12. Fiser DH, Tilford JM, Roberson PK. Relationship of illness severity and length of stay to functional outcomes in the pediatric intensive care unit: a multi-institutional study. Crit Care Med. 2000;28:1173-79.

13. Zaritsky A, Nadkarni V, Hazinski MF, Foltin G, Quan L, Wright J, et al. Recommended guidelines for uniform reporting of pediatric advanced life support: the pediatric Utstein style. A statement for healthcare professionals from a task force of the American Academy of Pediatrics, the American Heart Association, and the European Resuscitation Council. Pediatrics. 1995;96(4 Pt 1):765-79.

14. Morris MC, Wernovsky G, Nadkarni VM. Survival outcomes after extracorporeal cardiopulmonary resuscitation instituted during active chest compressions following refractory in-hospital pediatric cardiac arrest. Pediatr Crit Care Med. 2004;5:440-6.

15. Perez A, Minces PG, Schnitzler EJ, Agosta GE, Medina SA, Ciraolo CA. Jugular venous oxygen saturation or arteriovenous difference of lactate content and outcome in children with severe traumatic brain injury. Pediatr Crit Care Med. 2003;4:33-8.

16. Spinella PC, Dominguez T, Drott HR, Huh J, McCormick L, Rajendra A, et al. S-100beta protein-serum levels in healthy children and its association with outcome in pediatric traumatic brain injury. Crit Care Med. 2003;31:939-45.

17. Martha VF, Garcia PC, Piva JP, Einloft PR, Bruno F, Rampon V. Comparação entre dois escores de prognóstico (PRISM e PIM) em unidade de terapia intensiva pediátrica. J Pediatr (Rio J). 2005;81:259-64.

18. Sperber AD. Translation and validation of study instruments for cross-cultural research. Gastroenterology. 2004;126(1 Suppl 1): S124-8. 
19. Volakli EA, Sdouga M, Tamiolaki M, Antoniades A, Reizoglou M, Giala MM. Mortality and morbidity two years after pediatric intensive care. Intensive Care Med. 2004;30 Suppl 1: S127.

20. Cunha F, Carvalho L, Pereira G, Santo E, Ribeiro O, Santos LA. Estado funcional de los niños ingresados en unidades de cuidados intensivos pediátricos portuguesas. In: Reunión Nacional, Sociedad Española de Cuidados Intensivos Pediátricos (SECIP) de la AEP 3; 2006 Nov 17-18; Madrid, España. http:// reunir.med.up.pt. Access: 21/01/2007.

21. Taylor A, Butt W, Ciardulli M. The functional outcome and quality of life of children after admission to an intensive care unit. Intensive Care Med. 2003;29:795-800.

22. Ruttimann UE, Pollack MM, Fiser DH. Prediction of three outcome states from pediatric intensive care. Crit Care Med. 1996;24:7885.
23. Eiser C. Children's quality of life measures. Arch Dis Child. 1997; 77:350-4.

24. Haley SM, Graham RJ, Dumas HM. Outcome rating scales for pediatric head injury. J Intensive Care Med. 2004;19:205-19.

Correspondence:

Patrícia Tollens Alievi

Av. Praia de Belas, 1870/405

CEP 90110-000 - Porto Alegre, RS - Brazil

Tel.: +55 (51) 3217.1003

E-mail: patitollens@bol.com.br 